\title{
ON THE SECOND HOMOTOPY MODULE OF TWO-DIMENSIONAL CW COMPLEXES
}

M. N. DYER

\begin{abstract}
Let $X$ be a connected 2-dimensional CW complex. This note reproves from a very simple point of view two classical theorems of $\mathbf{H}$. Hopf relating the homology of the fundamental group $\pi=\pi_{1}(X)$ of $X$ and the Hurewicz map on $\pi_{2}(X)$. This point of view also allows the dual theorems to be proved. If $\pi$ is a finite group, a new interpretation is given for $H_{i}(\pi ; Z)(i=2,3)$ in terms of $\pi_{2}(X)$.
\end{abstract}

1. Introduction. Let $\pi$ be an arbitrary group. A [ $\pi, 2]$-complex is any connected two-dimensional CW complex with fundamental group $\pi$. Let $X$ be a $[\pi, 2]$-complex. In this note we explore certain interesting relations involving the $\pi$-modules $\pi_{2}(X)$.

For example, we extend and dualize the theorems of H. Hopf [2], [8], [9]. Let $X$ be a $[\pi, 2]$-complex, $p: \tilde{X} \rightarrow X$ be the universal covering map, $h$ : $\pi_{2}(X) \rightarrow H_{2}(X)$ the Hurewicz homomorphism with spherical image $\Sigma_{2}(X)$, and $A=A(\pi)$, the augmentation ideal in the integral group ring $Z \pi$. If $M$ is a $\pi$-module, then $M^{\pi}$ is the submodule of elements fixed under the action of $\pi$ and $A M$ is the submodule generated by $\{a \cdot m \mid a \in A, m \in M\}$. If $\pi$ is a finite group and $M$ any $\pi$-module, let ${ }_{N} M=\operatorname{ker}\{\bar{N}: M \rightarrow M\}$ where $\bar{N}(m)$ $=N \cdot m$ and $N=\sum_{x \in \pi} x$ is the norm element in $Z \pi$.

1.1. TheOREM. Let $X$ be any [ $\pi, 2]$-complex. The following table expresses the theorem: $H_{*}^{(*)}(\pi)$ is the homology (cohomology) of the group $\pi$ with coefficients in the trivial $\pi$-module $Z . H_{*}^{(*)}(\tilde{X})$ is the cellular homology $($ cohomology) of the space $\tilde{X}$, considered as a $\pi$-module via the action of $\pi$ on $\tilde{X}$.
A. $\pi$ arbitrary
B. $\pi$ finite

$$
\begin{gathered}
H_{2}(X) / \Sigma_{2}(X) \cong H_{2}(\pi) \cong \pi_{2}(X)^{\pi} / N \cdot \pi_{2}(X) \\
\operatorname{ker} h / A \cdot \pi_{2}(X) \cong H_{3}(\pi) \cong{ }_{N} \pi_{2}(X) / A \cdot \pi_{2}(X) \\
\operatorname{ker}\left\{p^{*}: H^{2}(X) \rightarrow H^{2}(\tilde{X})\right\} \cong H^{2}(\pi) \cong{ }_{N} H^{2}(\tilde{X}) / A \cdot H^{2}(\tilde{X}) \\
H^{2}(\tilde{X})^{\pi} / \operatorname{im} p^{*} \cong H^{3}(\pi) \cong H^{2}(\tilde{X})^{\pi} / N \cdot H^{2}(\tilde{X})
\end{gathered}
$$

Note. The Hopf theorems are expressed as $1.1 \mathrm{~A}(\mathrm{a}),(\mathrm{b})$.

2. Arbitrary fundamental group. Given any two (left) $\pi$-modules $A, B$,

$$
A \otimes_{Z} B \equiv A \otimes B
$$

is the $\pi$-module with diagonal action given by $x(a \otimes b)=x a \otimes x b(x \in \pi$,

Received by the editors November 5, 1974.

AMS (MOS) subject classifications (1970). Primary 55A20, 55E99, $18 \mathrm{H} 10$.

(c) American Mathematical Society 1976 
$a \in A, b \in B$ ). For any connected [ $\pi, 2]$-complex $X$ and $\pi$-module $G$, let $H_{i}(X ; \mathbf{G})$ denote singular homology with local coefficients in $G$.

2.1. TheOREM. For any $[\pi, 2]$-complex $X$ and any $\pi$-module $G$,

$$
\begin{gathered}
H_{1}(X ; \mathbf{G}) \cong H_{1}(\pi ; G), \\
H_{i+3}(\pi ; G) \cong H_{i}\left(\pi ; \pi_{2}(X) \otimes G\right)(i \geqslant 1),
\end{gathered}
$$

(c) $0 \rightarrow H_{3}(\pi ; G) \rightarrow H_{0}\left(\pi ; \pi_{2}(X) \otimes G\right) \stackrel{\hbar}{\rightarrow} H_{2}(X ; \mathbf{G}) \rightarrow H_{2}(\pi ; G) \rightarrow 0$,

is an exact sequence of $\pi$-modules, where $\bar{h}$ is induced by the Hurewicz homomorphism [4].

With $G=Z$, the trivial $\pi$-module, 2.1(c) is equivalent to the theorems of $\mathrm{H}$. Hopf for $[\pi, 2]$-complexes given in $1.1 \mathrm{~A}(\mathrm{a}),(\mathrm{b})$.

If $G$ is an arbitrary $\pi$-module, then any module of the form $Z \pi \otimes G$ (with diagonal action) is called induced. If $F$ is any free $\pi$-module, then

$$
F \otimes G \cong\left(\underset{\lambda \in A}{\bigoplus_{\lambda}}(Z \pi)_{\lambda}\right) \otimes G \cong Z \pi \otimes\left(\bigoplus_{\lambda \in A} G_{\lambda}\right)
$$

is induced. A direct computation shows that $H_{i}(\pi ; Z \pi \otimes G)=0$ for any $i>0$ (see [7, p. 211]). Thus it is not surprising that if one [ $\pi, 2]$-complex $X$ satisfies 2.1(b), then so does any other [ $\pi, 2]$-complex $X^{\prime}$. This follows because a theorem of J.H.C. Whitehead implies that $\pi_{2}(X) \oplus F \cong \pi_{2}\left(X^{\prime}\right) \oplus$ $F^{\prime}$ for suitable free $\pi$-modules $F$ and $F^{\prime}$.

Proof. Let $\tilde{X}$ be the universal cover of $X$ and

$$
\begin{aligned}
\tilde{X} \rightarrow & X \\
& \downarrow \\
& \\
& K(\pi, 1)
\end{aligned}
$$

be the associated fibration (see [10, p. 286]). The Serre spectral sequence for local coefficients in $G$ has $E_{p, q}^{2} \cong H_{p}\left(\pi ; H_{q}(\tilde{X} ; G)\right)\left(H_{q}(\tilde{X} ; G)\right.$ is the $q$ th cellular homology of $\tilde{X}$ with (standard) coefficients in the underlying abelian group $G_{0}$ of $G$ and is considered a $\pi$-module via the diagonal action of $\pi$ on $C_{*}(\tilde{X}) \otimes G$ and converges to $\left.H_{*}(X ; \mathbf{G})\right)$. $\tilde{X}$ is simply connected and twodimensional yields $E_{p, q}^{2}=0$ unless $q=0$, 2. A long exact sequence thus arises [10, p. 240]:

$$
\begin{aligned}
\cdots & \rightarrow H_{i-2}\left(\pi ; \pi_{2}(X) \otimes G\right) \rightarrow H_{i}(X ; \mathbf{G}) \rightarrow H_{i}(\pi ; G) \\
& \stackrel{d_{3}}{\rightarrow} H_{i-3}\left(\pi ; \pi_{2}(X) \otimes G\right) \rightarrow \ldots \rightarrow H_{3}(X ; \mathbf{G}) \rightarrow H_{3}(\pi ; G) \\
& \stackrel{d_{3}}{\rightarrow} H_{0}\left(\pi ; \pi_{2}(X) \otimes G\right) \stackrel{h}{\rightarrow} H_{2}(X ; \mathbf{G}) \rightarrow H_{2}(\pi ; G) \rightarrow 0 .
\end{aligned}
$$

This, together with the fact that $X$ is 2-dimensional, yields the result. It is well known that if $p: \tilde{X} \rightarrow X$ is the projection, then $p_{\#}: H_{2}(\tilde{X}) \rightarrow H_{2}(X)$ is (essentially) the Hurewicz map. So with the identification

$$
\pi_{2}(X) \stackrel{p_{*}}{\longleftarrow} \pi_{2}(\tilde{X}) \stackrel{\tilde{h}}{\cong} H_{2}(\tilde{X})
$$

we have $\bar{h}$ is induced by $h: \pi_{2}(X) \rightarrow H_{2}(X)$, according to [10, p. 271]. 
SECOND PROOF of (b). For this we use the trick of W. Cockcroft and R. Swan [3] together with the fact that $H_{i}(\pi ; M)=0(i>0)$ for any induced module $M$. The [ $\pi, 2]$-complex $X$ gives rise to an exact sequence of $\pi$-modules

$$
e_{*}(\tilde{X}): 0 \rightarrow \pi_{2}(X) \rightarrow C_{2} \stackrel{\partial_{2}}{\rightarrow} C_{1} \stackrel{\partial_{1}}{\rightarrow} C_{0} \stackrel{\varepsilon}{\rightarrow} Z \rightarrow 0,
$$

where $e_{*}(\tilde{X})$ is the cellular chain complex for the universal $\tilde{X}, C_{i}$ is a free $\pi$-module with rank equal to the number of $i$-cells in $X$, and $\varepsilon$ is the augmentation homomorphism. This spawns three short exact sequences

$$
\begin{gathered}
0 \rightarrow \pi_{2}(X) \otimes G \rightarrow C_{2} \otimes G \rightarrow B_{1} \otimes G \rightarrow 0, \\
0 \rightarrow B_{1} \otimes G \rightarrow C_{1} \otimes G \rightarrow B_{0} \otimes G \rightarrow 0, \\
0 \rightarrow B_{0} \otimes G \rightarrow C_{0} \otimes G \rightarrow G \rightarrow 0 .
\end{gathered}
$$

Since $H_{i}\left(\pi ; C_{i} \otimes G\right)=0$ for $i>0$, the coefficient sequences for $H_{i}(\pi,-)$ associated with the above exact sequences give

$$
\begin{aligned}
& H_{i+1}\left(\pi, B_{1} \otimes G\right) \stackrel{\partial_{i+1}}{\rightrightarrows} H_{i}\left(\pi ; \pi_{2}(X) \otimes G\right), \\
& H_{i+2}\left(\pi, B_{0} \otimes G\right) \underset{\simeq}{\stackrel{\partial_{i+2}}{\longrightarrow}} H_{i+1}\left(\pi, B_{1} \otimes G\right), \\
& H_{i+3}(\pi, G) \stackrel{\partial_{i+3}}{\longrightarrow} H_{i+2}\left(\pi, B_{0} \otimes G\right) \quad(i \geqslant 1) .
\end{aligned}
$$

Then $\beta=\partial_{i+1} \partial_{i+2} \partial_{i+3}$ is the prescribed isomorphism. That $\beta=d_{3}$ in the spectral sequence is implied by the discussion in [11, p. 343].

For any two left $\pi$-modules $A, B$, define $\operatorname{Hom}(A, B) \equiv \operatorname{Hom}_{Z}(A, B)$ to be the $\pi$-module with diagonal action: $(x f)(a)=x f\left(x^{-1} a\right)(x \in \pi, f \in$ $\operatorname{Hom}(A, B), a \in A)$. We state the corresponding results for cohomology (with similar proof).

2.2. TheOREM. Let $X$ be $a[\pi, 2]$-complex, $G$ a $\pi$-module. Then

$$
\begin{gathered}
H^{1}(\pi ; G) \cong H^{1}(X ; \mathbf{G}), \\
\text { (b) } H^{i}\left(\pi ; H^{2}(\tilde{X} ; G)\right) \stackrel{\rightarrow}{\cong} H^{i+3}(\pi ; G)(i \geqslant 1), \\
\text { (c) } 0 \rightarrow H^{2}(\pi ; G) \rightarrow H^{2}(X ; \mathbf{G}) \stackrel{\bar{p}}{\rightarrow} H^{0}\left(\pi ; H^{2}(\tilde{X} ; G)\right) \rightarrow H^{3}(\pi ; G) \rightarrow 0
\end{gathered}
$$

is exact, where $\bar{p}$ is induced from $p: \tilde{X} \rightarrow X$. Again $H^{2}(\tilde{X} ; G)$ has untwisted coefficients in the underlying abelian group $G_{0}$ of $G$, and $H^{2}(\tilde{X} ; G)$ is considered $a \pi$-module via the diagonal action on $C^{*}(\tilde{X} ; G)=\operatorname{Hom}_{Z}\left(C_{*}(\tilde{X}), G\right)$.

Note. For $G=Z, 2.2(\mathrm{c})$ yields Theorem (1.1) A(c), (d). The use of the Cockcroft-Swan technique to prove 2.2(b) is possible because for any free $\pi$-module $F=\bigoplus_{\lambda \in \Lambda}(Z \pi)_{\lambda}, \operatorname{Hom}_{Z}(F ; G)$ is coinduced; that is to say,

$$
\operatorname{Hom}(F, G) \cong \operatorname{Hom}\left(Z \pi, \prod_{\lambda \in \Lambda}(G)_{\lambda}\right)
$$

as $\pi$-modules. This implies that $H^{i}(\pi ; \operatorname{Hom}(F ; G))=0$ for $i>0$ [7, p. 211].

3. Finite fundamental group. For $\pi$ finite we use the Tate cohomology $\hat{H}^{i}(\pi ;-)(i \in Z)[1$, Chapter 11] given by 


$$
\hat{H}^{i}(\pi ; G)= \begin{cases}H^{i}(\pi ; G) & \text { if } i>0, \\ G^{\pi} / N \cdot G & \text { if } i=0, \\ N^{G} / A \cdot G & \text { if } i=-1, \\ H_{-i-1}(\pi ; G) & \text { if } i<0,\end{cases}
$$

where ${ }_{N} G=\operatorname{ker}\{\bar{N}: G \rightarrow G\}$ and $A \cdot G$ is the submodule generated by $\{a \cdot g \mid a \in A(\pi), g \in G\}$.

3.1. THEOREM. Let $\pi$ be a finite group and $G$ an arbitrary $\pi$-module. If $X$ is an $[\pi, 2]$-complex, then

(i) $\hat{H}^{t}(\pi ; G) \cong \hat{H}^{t+3}\left(\pi ; \pi_{2}(X) \otimes G\right)(t \in Z)$,

(ii) $\hat{H}^{t}(\pi ; G) \cong \hat{H}^{t-3}\left(\pi ; H^{2}(\tilde{X} ; G)\right)(t \in Z)$.

Proof. Apply the Cockcroft-Swan technique to the exact sequences

$$
0 \rightarrow \pi_{2}(X) \rightarrow C_{2}(\tilde{X}) \rightarrow C_{1}(\tilde{X}) \rightarrow C_{0}(\tilde{X}) \rightarrow Z \rightarrow 0
$$

and

$$
\begin{aligned}
0 & \rightarrow Z \rightarrow \operatorname{Hom}\left(C_{0}, G\right) \rightarrow \operatorname{Hom}\left(C_{1}, G\right) \\
& \rightarrow \operatorname{Hom}\left(C_{2}, G\right) \rightarrow H^{2}(\tilde{X} ; G) \rightarrow 0 .
\end{aligned}
$$

In order to apply this, one needs to use the fact that $\hat{H}^{*}(\pi ; F)=0$ for $F$ a free $\pi$-module [1, p. 199] or $F=\operatorname{Hom}\left(F^{\prime}, G\right)$, where $F^{\prime}$ is free.

Note. 3.1 proves Theorem $1.1 \mathrm{~B}(\mathrm{a})-(\mathrm{d})$ simply by letting $G$ be the trivial $\pi$-module $Z$ and by setting $t$ equal to the following values:

\begin{tabular}{c|r} 
(1.1)B & $t$ equals \\
\hline (a) & -3 in (i) \\
(b) & -4 in (i) \\
(c) & 2 in (ii) \\
(d) & 3 in (ii)
\end{tabular}

Other values of $t$ lead to interesting information:

(e) $H^{2}\left(\pi ; \pi_{2}(X)\right) \cong \hat{H}^{-1}(\pi)=0(t=-1$ in (i) )

(f) $H_{3}\left(\pi ; H^{2}(\tilde{X})\right) \cong \hat{H}^{-1}(\pi)=0(t=-1$ in (ii)),

(g) $H^{1}\left(\pi ; \pi_{2}(X)\right) \cong H_{1}(\pi) \cong$ abelianization of $\pi(t=-2$ in (i)).

(e), of course, is a result of A. J. Sieradski [5] and allows one to show that, for $X$ a $[\pi, 2]$-complex, the self-equivalence group $\varepsilon(X)$ of homotopy classes of homotopy equivalences $X \rightarrow X$ is isomorphic to Aut $T(X)$, the group of automorphisms of the algebraic two-type $T(X)$ of $X$ [5].

Finally, 3.1 has the following amusing corollary.

3.2. Corollary. Let $\pi$ be a finite group. If $\pi$ admits a [ $\pi, 2]$-complex such that $H^{2}(\tilde{X}) \cong \pi_{2}(X)$ as $\pi$-modules, then $\hat{H}^{i}(\pi) \cong \hat{H}^{i+6}(\pi)(i \in Z)$. Thus $\pi$ is a periodic group of period 2 or 6.

For example, if $X$ is any finite $\left[Z_{n}, 2\right]$-complex, then $H^{2}(\tilde{X}) \cong \pi_{2}(X)$ as a $Z_{n}$-module [6].

4. Generalizations. In this section we point out that these theorems, suitably interpreted, hold in much greater generality. The proofs are essentially the same. 
(a) All the results of this paper may be interpreted for $[\pi, m]$-complexes: connected, $m$-dimensional $\mathrm{CW}$ complexes such that $\pi_{1}(X)=\pi$ and for which $\tilde{X}$ is $(m-1)$-connected $(m \geqslant 2)$. Thus, for example, if $\pi$ is finite and $X$ is any $[\pi, m]$-complex, then

$$
\hat{H}^{t}(\pi ; G) \cong \hat{H}^{t+m+1}\left(\pi ; \pi_{m}(X) \otimes G\right) \quad(t \in Z) .
$$

(b) Even further, the results hold for quasi $[\pi, m]$-complexes: connected $\mathrm{CW}$ complexes such that $\pi_{1}(X)=\pi, \tilde{X}$ is $(m-1)$-connected, and the $\pi$ module $C_{m}(\tilde{X}) / B_{m}(\tilde{X})$ is projective [4]. As is seen in [5, Theorem 1.3], $X$ is a quasi $[\pi, m]$-complex iff $X$ has the topological $m$-type of a space dominated by a $[\pi, m]$-complex. If $m \geqslant 3$, then $X$ is a quasi $[\pi, m]$-complex iff $X$ has the $m$-type of a $[\pi, m]$-complex.

\section{BIBLIOGRAPHY}

1. H. Cartan and S. Eilenberg, Homological algebra, Princeton Univ. Press, Princeton, N. J., 1956. MR 17, 1040.

2. W. Cockcroft, On two-dimensional aspherical complexes, Proc. London Math. Soc. (3) 4 (1954), 375-384. MR 16, 62.

3. W. Cockcroft and R. G. Swan, On the homotopy type of certain two-dimensional complexes, Proc. London Math. Soc. (3) 11 (1961), 194-202. MR 23 \# A3567.

4. M. Dyer, On the 2-realizability of 2-types, Trans. Amer. Math. Soc. 204 (1975), 229-243.

5. The homotopy theory of $(\pi, m)$-complexes, J. Pure Appl. Algebra (to appear).

6. M. Dyer and A. J. Sieradski, Trees of homotopy types of two-dimensional CW complexes, Comment Math. Helv. 48 (1973), 31-44.

7. P. J. Hilton and U. Stammbach, A course in homological algebra, Graduate Texts in Math., vol. 4, Springer-Verlag, New York, 1972.

8. H. Hopf, Über die Bettischen Gruppen, die zu einer beliebigen Gruppe gehören, Comment. Math. Helv. 17 (1944), 39-79. MR 6, 279.

9. __ Beitrage zur Homotopietheorie, Comment. Math. Helv. 17 (1944), 307-326. MR 7, 36.

10. S.-T. Hu, Homotopy theory, Pure and Appl. Math., vol. 8, Academic Press, New York and London, 1959. MR 21 \#5186.

11. S. Mac Lane, Homology, Die Grundlehren der math. Wissenschaften, Band 114, Academic Press, New York; Springer-Verlag, Berlin, 1963. MR 28 \# 122.

Department of Mathematics, University of Oregon, Eugene, Oregon 97403 